\title{
Comparison of BISAP and MCTSI for Predicting the Severity of
}

\section{Hyperlipidemic Acute Pancreatitis}

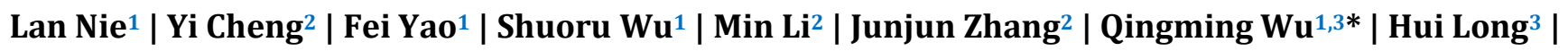 Xiangming Fang ${ }^{4}$}

\section{*Correspondence: Qingming Wu}

Address: ${ }^{1}$ Wuhan University of Science and Technology School of Medicine, Wuhan, Hubei, China; ${ }^{2}$ The General Hospital of Central Theater Command, Wuhan, Hubei, China; ${ }^{3}$ Tianyou Hospital Affiliated to Wuhan University of Science and Technology, Wuhan, Hubei, China; ${ }^{4}$ Puren Hospital Affiliated to Wuhan University of Science and Technology, Wuhan, Hubei, China e-mail $\bowtie$ : wuhe9224@sina.com

Received: 05 May 2020; Accepted: 19 May 2020

Copyright: (C) 2020 Nie L. This is an open-access article distributed under the terms of the Creative Commons Attribution License, which permits unrestricted use, distribution, and reproduction in any medium, provided that the original work is properly cited.

\section{ABSTRACT}

With the incidence of hyperlipidemic acute pancreatitis (HLAP) rapidly increasing, it is important for clinicians to identify the severity of HLAP at early stage. Several multifactorial scoring systems have been considered as accurate methods for risk stratification. Our goal was to compare the accuracy of bedside index for severity in acute pancreatitis (BISAP) and modified CT severity index (MCTSI) in predicting the prognosis of HLAP. Demographic characteristics were retrospectively analysed. Predictive accuracy of BISAP and MCTSI was investigated by Area Under the Curve (AUC) based on the receiver operating characteristic (ROC) curve. A total of 167 patients with HLAP were included. 133 of patients were classified as Mild acute pancreatitis (MAP), and 34 were Moderately severe acute pancreatitis (MSAP) and Severe acute pancreatitis (SAP). Compared with MAP group, the level of Triglycerides (TG) [(23.3 \pm 11.9$)$ vs $(13.1 \pm 8.2) \mathrm{mmol} / \mathrm{L}, \mathrm{P}<0.01]$ and Total cholesterols (TC) $[(12.0 \pm 8.9)$ vs $(8.5 \pm 4.0) \mathrm{mmol} / \mathrm{L}, \mathrm{P}=0.03]$ were higher in MSAP and SAP group, the incidence of fatty liver (82.4\% vs $50.4 \%$, $\mathrm{p}=0.01)$ and local complications $(73.5 \%$ vs $29.3 \%, \mathrm{p}=0.01)$ were also higher. The AUC of BISAP to predict severe HLAP and local complications respectively were $0.89,0.73$, MCTSI were $0.78,0.87$. Compared to MCTSI, BISAP may be a better prognostic scoring system for predicting the severity of HLAP in view of accuracy and easiness.

Keywords: Hyperlipidemic Acute Pancreatitis, Triglycerides, BISAP Score, MCTSI Score, Severe Acute Pancreatitis

\section{Introduction}

Acute Pancreatitis (AP) is an acute inflammatory disorder with varying etiology. The disease was grouped into Mild acute pancreatitis (MAP), Moderately severe acute pancreatitis (MSAP) and Severe acute pancreatitis (SAP) based on Atlanta 2012 definitions. The overall mortality rate was about 5\% $10 \%$, but 36\% 50\% in patients with SAP (Banks et al., 2013). In Western countries, gallstones and alcohol are two predominant etiological factors of AP, followed by hypertriglyceridemia (HTG) as the third most common cause. In China, HTG has higher proportions than alcohol of AP causes and becomes the second most common etiology (Fan et al., 2018). Hyperlipidemic acute pancreatitis (HLAP) had a 
complex pathophysiology and likely potentially relapsed (Zheng et al., 2015).

So far, there are a large number of scoring systems to predict the severity and complications of AP, such as Ranson,s score, the Acute Physiology and Chronic Health Evaluation (APACHE II) score, BISAP score, MCTSI score, et al (Mortele et al., 2004; Wu et al., 2008; Ranson et al., 1976; Knaus et al., 1981). Nevertheless no universally adopted standards are formulated. Modified CT severity index (MCTSI) was formed based on CT severity index (CTSI), which not only reflects pancreatic inflammation and necrosis but also organ failure and extrapancreatic complications (Mortele et al., 2004). Bedside index for severity in acute pancreatitis (BISAP) score is a simple scoring system initially proposed by Wu (Wu et al., 2008) in 2008. When BISAP $\geq 3$, it was expected to be MSAP and SAP and the mortality rate markedly increased. BISAP and MCTSI scores are welcomed among clinicians because of inexpensive, based on routine and easily obtained.

The purposes of our study were to primarily evaluate the predicting capability of BISAP as a early scoring system and compared with MCTSI.

\section{Methods}

\section{Data collection}

We enrolled 167 patients with HLAP over the duration of January 2017 to December 2018 in three representative general hospitals. The exclusion criterias included patients with malignant tumour, pregnancy and transferred from other hospital. eleven patients were absent from further analysis for imaging and laboratory information missing.

The diagnosis of AP demands at least two of the following three characteristics: 1) abdominal pain consistent with acute pancreatitis (acute onset of a persistent, severe, epigastric pain often radiating to the back); 2) serum lipase activity (or amylase activity) at least three times greater than the upper limit of normal; and 3) characteristic findings of acute pancreatitis on computed tomography (CT) and less commonly magnetic resonance imaging (MRI) or transabdominal ultrasonography. In addition to above features, HLAP was diagnosed if TG met $11.0 \mathrm{mmol} / \mathrm{L}$, or ranged from 5.56 to $11.0 \mathrm{mmol} / \mathrm{L}$ but accompanied with chylemia, except for other etiologies of AP, namely, gallstone, drug, infection, et al. The category of severity (MAP, MSAP, SAP) and local complications (acute peripancreatic fluid collection, pancreatic pseudocyst, acute necrotic collection and walled-off necrosis) were in accordance with 2012 revised Atlanta criteria.

BISAP score contained (Wu et al., 2008): 1) blood urea nitrogen (BUN) >25 mg/dl; 2) impaired 
mental status; 3) systemic inflammatory response syndrome (SIRS); 4) age >60 years; 5) or the presence of a pleural effusion, which were calculated within the first $24 \mathrm{~h}$ after admission (each one represents 1 point).

The indicator of MCTSI (Mortele et al., 2004): 1) Pancreatic inflammation: Normal pancreas 0 point, Intrinsic pancreatic abnormalities with or without inflammatory changes in peripancreatic fat 2 points,Pancreatic or peripancreatic fluid collection or peripancreatic fat necrosis 4 points; 2) Pancreatic necrosis: None 0 point, $\leq 30 \% 2$ points, $>30 \% 4$ points; and 3) Extrapancreatic complications (one or more of pleural effusion, ascites, vascular complications, parenchymal complications, or gastrointestinal tract involvement) 2 points, were collected within one week of onset of symptoms and evaluated by two radiologists and one clinicians.

\section{Statistical analysis}

SPSS 24.0 software was used for statistical analysis. Descriptive data were performed as mean and standard deviation, Categorical data as frequencies and percentages. Groups comparison used Student's ttest or Mann-Whitney Test for Descriptive data and Chi-square or Fishers, exact test for Categorical data. Correlation analysis was demonstrated by Spearman's test. ROC curve was drawn corresponding AUC and sensitivity, specificity, positive predictive value (PPV), negative predictive value (NPV), Youden index computed. $\mathrm{P}<0.05$ was considered statistically significant.

Results

As showing in Table 1, Table 2, Table 3. Of 167 patients with HLAP, 133 patients (79.6\%) were diagnosed as MAP, 34 (20.3\%) were MSAP and SAP. The median ages were $39.8 \pm 11.0$ years old with 90 male $(67.7 \%)$ in MAP group and $39.7 \pm 11.0$ years old with 24 male $(70.6 \%)$ in MSAP and SAP group. There was no statistical significance between two groups in age and gender $(\mathrm{P}>0.05)$. Compared with MAP group, The level of Triglycerides $(\mathrm{TG})[(23.3 \pm 11.9)$ vs $(13.1 \pm 8.2) \mathrm{mmol} / \mathrm{L}, \mathrm{P}<0.01]$ and Total cholesterols $(\mathrm{TC})[(12.0 \pm 8.9)$ vs $(8.5 \pm 4.0) \mathrm{mmol} / \mathrm{L}, \mathrm{P}=0.03]$ were higher in MSAP and SAP group, the incidence of fatty liver (82.4\% vs $50.4 \%, \mathrm{p}=0.01$ ) and local complications (73.5\% vs $29.3 \%, \mathrm{p}=0.01$ ) were also higher, the length of hospital days $[(13.0 \pm 4.8)$ vs $(8.3 \pm 2.7) \mathrm{mmol} / \mathrm{L}, \mathrm{P}<0.01]$ were longer. The severity of HLAP increased as the BISAP and MCTSI scores increased $(\mathrm{P}<0.01)$. No differences were detected between two groups in Alanine aminotransferase (ALT), Aspertate aminotransferase (AST), and blood amylase $(\mathrm{P}>0.05)$. The underlying comorbidities including Hypertension, Hyperuricemia, Type 2 diabete showed no statistical significance $(\mathrm{P}>0.05)$. 
Table 1: Comparison of clinical features between two groups.

\begin{tabular}{|c|c|c|c|}
\hline Features & MAP & MSAP and SAP & $P$ value \\
\hline & $n=133$ & $\mathrm{n}=34$ & \\
\hline \multicolumn{4}{|l|}{ Gender, \% } \\
\hline Male & $90(67.7)$ & $24(70.6)$ & 0.74 \\
\hline Female & $43(32.3)$ & $10(29.4)$ & \\
\hline Age, years & $39.8 \pm 11.0$ & $39.7 \pm 11.0$ & 0.95 \\
\hline ALT (U/L) & $29.1 \pm 20.0$ & $27.0 \pm 20.1$ & 0.59 \\
\hline AST (U/L) & $27.0 \pm 20.1$ & $32.1 \pm 31.8$ & 0.49 \\
\hline TG (mmol/L) & $13.1 \pm 8.2$ & $23.3 \pm 11.9$ & $<0.01$ \\
\hline $\mathrm{TC}(\mathrm{mmol} / \mathrm{L})$ & $8.5 \pm 4.0$ & $12.0 \pm 8.9$ & 0.03 \\
\hline Amylase (U/L) & $304.7 \pm 336.7$ & $349.2 \pm 306.2$ & 0.46 \\
\hline BISAP & $0.9 \pm 0.8$ & $2.5 \pm 0.9$ & $<0.01$ \\
\hline MCTSI & $2.9 \pm 1.4$ & $4.8 \pm 1.8$ & $<0.01$ \\
\hline Hospitalization days & $8.3 \pm 2.7$ & $13.0 \pm 4.8$ & $<0.01$ \\
\hline \multicolumn{4}{|l|}{ Local complication, \% } \\
\hline 0 & $94(70.7)$ & $9(26.5)$ & $<0.01$ \\
\hline 1 & $39(29.3)$ & $25(73.5)$ & \\
\hline \multicolumn{4}{|l|}{ Hypertension, \% } \\
\hline 0 & 105 (78.9) & $24(70.6)$ & 0.3 \\
\hline 1 & $28(21.1)$ & $10(29.4)$ & \\
\hline \multicolumn{4}{|l|}{ Hyperuricemia, \% } \\
\hline 0 & $106(79.7)$ & $30(88.2)$ & 0.25 \\
\hline 1 & $27(20.3)$ & $4(11.8)$ & \\
\hline \multicolumn{4}{|l|}{ Type 2 diabete, $\%$} \\
\hline 0 & $87(65.4)$ & $21(61.8)$ & 0.69 \\
\hline 1 & $46(34.6)$ & $13(38.2)$ & \\
\hline \multicolumn{4}{|l|}{ Fatty liver, \% } \\
\hline 0 & $66(49.6)$ & $6(17.6)$ & 0.01 \\
\hline 1 & $67(50.4)$ & $28(82.4)$ & \\
\hline
\end{tabular}

Table 2: Analysis between BISAP and MCTSI in predicting severity of HLAP.

\begin{tabular}{|c|c|c|c|}
\hline Scoring system & MAP & MSAP and SAP & P value \\
\hline & $\mathrm{n}=133$ & $\mathrm{n}=34$ & \\
\hline BISAP & & & \\
$\geq 3$ & $6(4.5 \%)$ & $25(73.5 \%)$ & $<0.01$ \\
\hline$<3$ & $127(95.5 \%)$ & $9(26.5 \%)$ & \\
\hline MCTSI & & & \\
$\geq 4$ & $53(39.8 \%)$ & $27(79.4 \%)$ & $<0.01$ \\
\hline$<4$ & $80(60.2 \%)$ & $7(20.6 \%)$ & \\
\hline
\end{tabular}


Table 3: Analysis between BISAP and MCTSI in predicting local complication of HLAP.

\begin{tabular}{|c|c|c|c|}
\hline Scoring system & No Local complication & Local complication & P value \\
\hline & $\mathrm{n}=103$ & $\mathrm{n}=64$ & \\
\hline BISAP & & & \\
$\geq 3$ & $7(6.8 \%)$ & $24(37.5 \%)$ & $<0.01$ \\
\hline$<3$ & $96(93.2 \%)$ & $40(62.5 \%)$ & \\
\hline MCTSI & & & \\
\hline 44 & $22(21.4 \%)$ & $58(90.6 \%)$ & $<0.01$ \\
\hline$<4$ & $81(78.6 \%)$ & $6(9.4 \%)$ & \\
\hline
\end{tabular}

As seen in Table 4, Fig. 1-2. The AUC for BISAP and MCTSI predicting severe HLAP respectively were $0.89,0.78$, sensitivity were $73.5 \%, 79.4 \%$, specificity were $95.5 \%, 60.2 \%$, PPV were $80.6 \%, 33.8 \%$, NPV were 93.4\%, 92.0\%. In predictionig local complications, the AUC of BISAP and MCTSI respectively were $0.73,0.87$, sensitivity were $37.5 \%, 90.1 \%$, specificity were $93.2 \%, 78.6 \%$, PPV were $77.4 \%, 72.5 \%$, NPV were $70.6 \%, 93.1 \%$.

Table 4: Comparison between BISAP and MCTSI in predicting severity and local complications of HLAP.

\begin{tabular}{|l|l|l|l|l|l|l|}
\hline Scoring system & sensitivity (\%) & specificity (\%) & PPV (\%) & NPV (\%) & Youden index & AUC \\
\hline MSAP and SAP & & & & & & \\
\hline BISAP & 73.5 & 95.5 & 80.6 & 93.4 & 0.69 & 0.89 \\
\hline MCTSI & 79.4 & 60.2 & 33.8 & 92 & 0.4 & 0.78 \\
\hline Local complication & & & & & & \\
\hline BISAP & 37.5 & 93.2 & 77.4 & 70.6 & 0.31 & 0.73 \\
\hline MCTSI & 90.1 & 78.6 & 72.5 & 93.1 & 0.69 & 0.87 \\
\hline
\end{tabular}

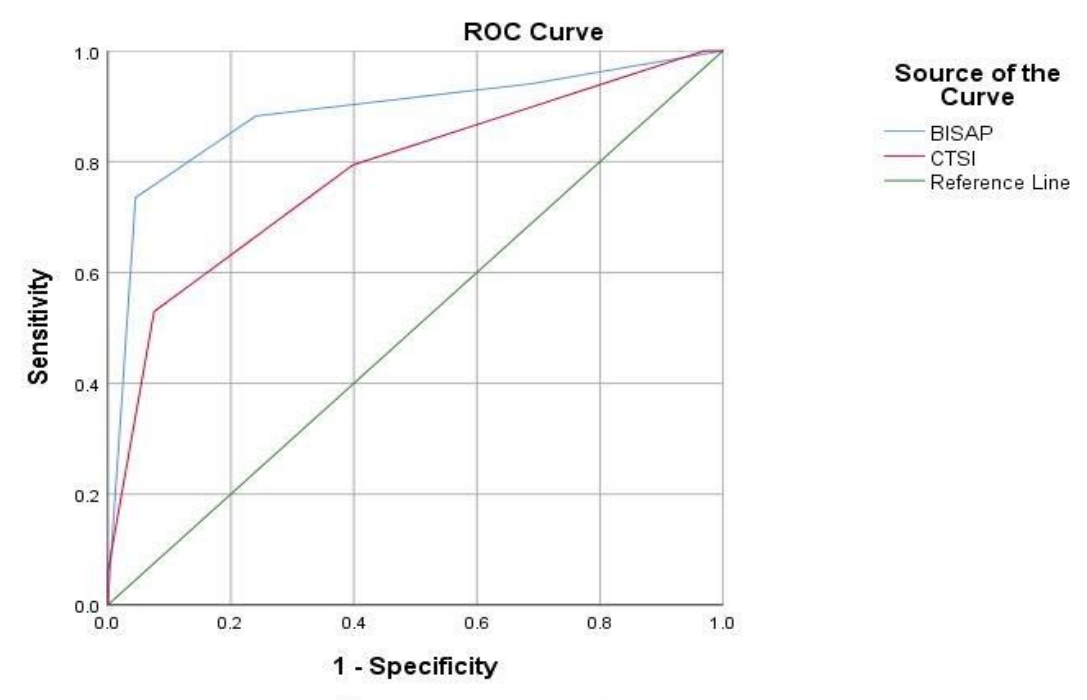

Figure 1: ROC curve of BISAP and MCTSI about the severity of HLAP. 

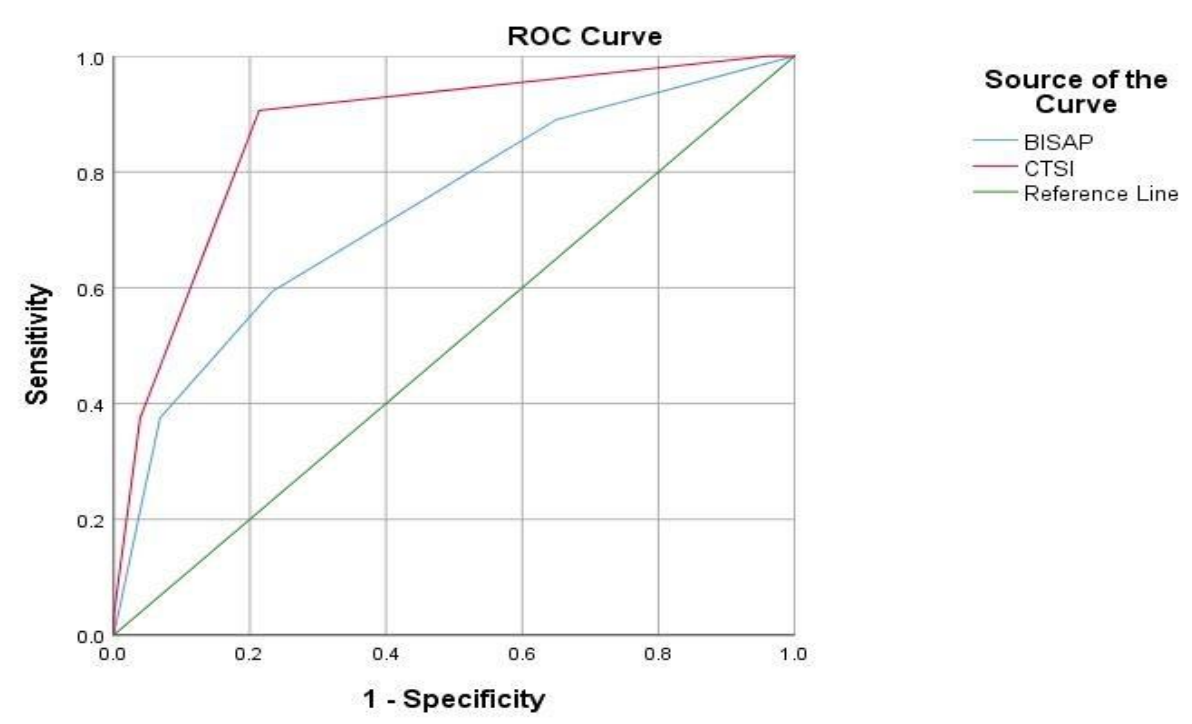

Figure 2: ROC curve of BISAP and MCTSI about local complications of HLAP.

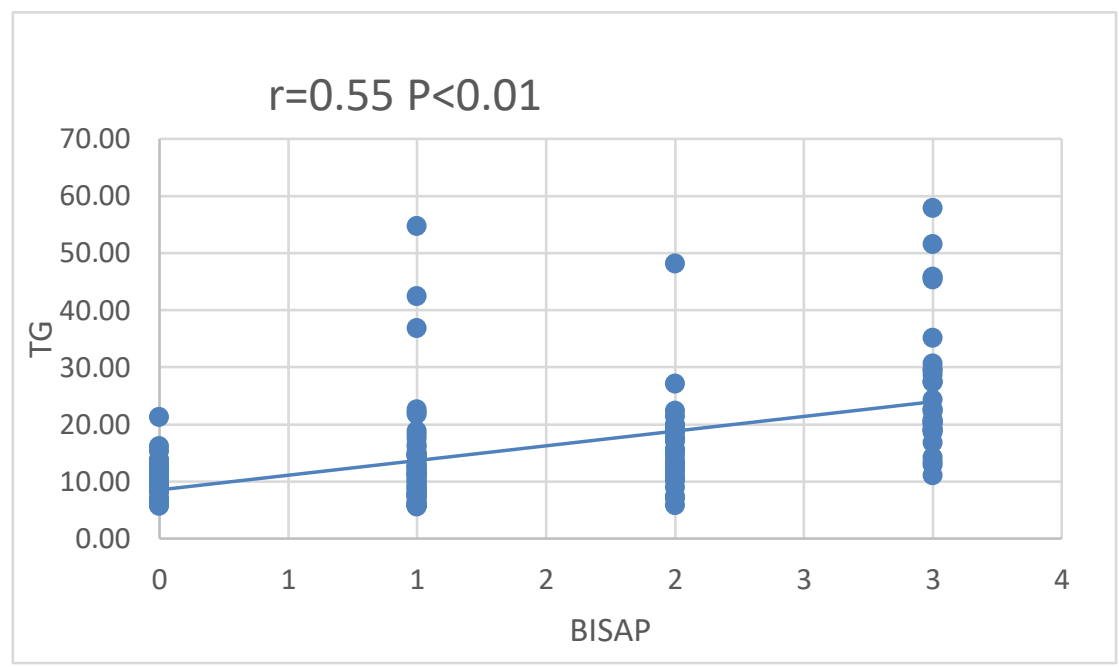

Figure 3: Correlation between BISAP and TG of HLAP.

\section{Discussion}

In 1952, Klatskin (Klatskin et al., 1952) initially proposed that hyperlipidemia may be the underlying cause for AP. It was widely known that gallstones (up to 60\%) and excess alcohol consumption (30\%) were the leading contributing factors of AP, while hyperlipidemia (HTG) was less frequency, accounting for 4-10\% (Adiamah et al., 2018). Nowadays excess high-sugar and high-fat food intake, overweight, and absent regular physical exercise bring about the elevated incidence for HLAP. A study about the trend for etiological distribution of AP during the period of five years in Beijing by Zheng et al (Zheng et al., 2015), found that the proportion of patients with HLAP was increasing year by year and HTG had taken place of the alcohol as the second common factors of AP. Compared to other 
etiological AP, HLAP had more complicated pathological mechanism, faster aggravation, easier relapse, higher mortality rate as high as 30\% (Valdivielso et al., 2014). Therefore, it is imperative to understand the etiology and predict the severity at early stage for better individualized treatment and management.

Our study highlighted that there were more man patients than woman patients with HLAP [(67.7\% vs $32.3 \%$ ) in MAP, (70.6\% vs 29.4\%) in MSAP and SAP] with the similar ages [39.8 \pm 11.0 in MAP, $39.7 \pm$ 11.0 in MSAP and SAP), which was in line with the report of Li, et al. (Li et al., 2018). These outcomes above probably were responsible for strong work and life pressure, smoking, absent exercise, highcalorie food, and irregular sleeping schedule in young man patients. Additionly, TG in MSAP and SAP group was higher than in MAP group $[(23.3 \pm 11.9)$ vs $(13.1 \pm 8.2) \mathrm{mmol} / \mathrm{L}, \mathrm{P}<0.01]$. Consistently, an analysis carried by Wang, et al. (Wang et al., 2016) indicated that when TG $>20.0 \mathrm{mmol} / \mathrm{L}$, MSAP and SAP patients accounted for 63.1\%, which was greatly higher than MAP petients. Oh, et al (Oh et al., 2007) and Yadav, et al. (Yadav et al., 2003) believed that the occurrence and mortality rate of AP would decrease when TG was controlled under $5.65 \mathrm{mmom} / \mathrm{L}$. A study concluded (Xiao et al., 2012) that patients with fatty liver was 59\% in MAP group and 100\% in MSAP and SAP group. Similar to previous study, the proportion of fatty liver in our research were 50.4\% in MAP group and $82.4 \%$ in MSAP and SAP group respectively.

BISAP was derived on data collected from 17992 cases of AP from 212 hospitals in 2000-2001 and validated by 18256 AP cases from 177 hospitals in 2004-2005. Wu et al concluded that BISAP was as effective as APACHE II to evaluate in-hospital mortality (Wu et al., 2008). Prior research demonstrated that the accuracy of BISAP for severity assessment of AP were not worse than "traditional" scoring systems (Ranson score, APACHE II, MCTSI, et al.) (G. I. Papachristou et al., 2010). A study by Qiu, et al. (Qiu et al., 2015) indicated that the AUC (0.604), sensitivity (0.905), and specificity (1.000) of BISAP were higher in the prediction of SAP compared to MCTSI. In our study, BISAP also performed better in predicting the severity of HLAP than MCTSI. Moreover, we found that BISAP was positive correlation with TG, both of which were important prognostic marker for HLAP.

MCTSI is a major clinical imaging scoring system showed strongly correlation with morbidity and local complications of AP (Aoun et al., 2009; Balthazar et al., 2002). It was modified and simplified based on CTSI that was put forward by Balthazar and colleagues (Mortele et al., 2004; Balthazar and Robinson et al., 1990). A study revealed that MCTSI manifested higher accuracy for the local complications and death of HLAP patients compared with nonhyperlipidemic acute pancreatitis (NHLAP) (G. I. Papachristou et al., 2010). According to the retrospective research from Yang, et al. (Yang et al., 2016), MCTSI had outstanding performance in the prediction of local complications but poor in severity assessment compared to BISAP. That conformed to our study. 
There were two limitations of the study. Firstly, the study was respective with small sample. Secondly, lack of comparison with other else traditional scoring systems.

\section{Conclusions}

Our study demonstrated that the level of TG and the incidence of fatty liver were higher in severer patients. TG was positively correlated with BISAP. Furthermore, BISAP may be a useful prognostic marker to predict the severity of AP.

\section{Author Contributions}

L N conceived the study; L N and QM W participated in the design and drafted the manuscript;L N, SR W, F Y, XM F, H L and Y C collected the data and performed statistical analyses.QM W, Y C, M L and JJ Z edited and checked the manuscript. All of the authors have read and approved the final manuscript.

\section{References}

Adiamah A, Psaltis E, Crook M. A systematic review of the epidemiology, pathophysiology and current management of hyperlipidaemic pancreatitis. Clin Nutr 2018; 37: 1810-1822.

Aoun E, Chen J, Reighard D, Gleeson FC, Whitcomb DC, Papachristou GI. Diagnostic accuracy of interleukin-6 and interleukin-8 in predicting severe acute pancreatitis: a meta-analysis. Pancreatology 2009; 9: 777-785.

Balthazar EJ, Robinson DL, Megibow AJ, Ranson JH. Acute pancreatitis: value of CT in establishing prognosis. Radiology $1990 ; 174: 331-336$.

Balthazar EJ. Acute pancreatitis: assessment of severity with clinical and CT evaluation. Radiology 2002; 223: 603-613.

Banks PA, Bollen TL, Dervenis C, Gooszen HG, Johnson CD, Sarr MG, Tsiotos GG, Vege SS. Classification of acute pancreatitis-2012: revision of the Atlanta classification and definitions by international consensus. Gut 2013; 62: $102-111$.

Fan J, Ding L, Lu Y, Zheng J, Zeng Y, Huang C. Epidemiology and Etiology of Acute Pancreatitis in Urban and Suburban Areas in Shanghai: A Retrospective Study. Gastroenterol Res Pract 2018; 2018: 1420590.

Klatskin G. Relationship between relapsing pancreatitis and essential hyperlipemia. Am J Med 1952; 12: 3-23.

Knaus WA, Zimmerman JE, Wagner DP, Draper EA, Lawrence DE. APACHE-acute physiology and chronic health evaluation: a physiologically based classification system. Crit Care Med 1981; 9: 591-597.

Li X, Ke L, Dong J, Ye B, Meng L, Mao W, Yang Q, Li W, Li J. Significantly different clinical features between hypertriglyceridemia and biliary acute pancreatitis: a retrospective study of 730 patients from a tertiary center. $B M C$ Gastroenterol 2018; 18: 1-89.

Mortele KJ, Wiesner W, Intriere L, Shankar S, Zou KH, Kalantari BN, Perez A, VanSonnenberg E, Ros PR, Banks PA, Silverman SG. A modified CT severity index for evaluating acute pancreatitis: improved correlation with patient outcome. AJR Am J Roentgenol 2004; 183: 1261-1265.

Oh RC and Lanier JB. Management of hypertriglyce ridemia. Am Fam Physician 2007; 75: 1365-1371. 
Papachristou GI, Muddana V, Yadav D, O'Connell M, Sanders MK, Slivka A, Whitcomb DC. Comparison of BISAP, Ranson's, APACHE-II, and CTSI scores in predicting organ failure, complications, and mortality in acute pancreatitis. Am J Gastroenterol $2010 ; 105: 435-441$.

Qiu L, Sun RQ, Jia RR, Ma XY, Cheng L, Tang MC, Zhao Y. Comparison of Existing Clinical Scoring Systems in Predicting Severity and Prognoses of Hyperlipidemic Acute Pancreatitis in Chinese Patients: A Retrospective Study. Medicine (Baltimore) 2015; 94: e957.

Ranson J, Rifkind KM, Turner JW. Prognostic signs and nonoperative peritoneal lavage in acute pancreatitis. Surg Gynecol Obstet 1976; 143: 209 - 219

Valdivielso P, Ramírez-Bueno A, Ewald N. Current knowledge of hypertriglyceridemic Pancreatitis. Eur J Intern Med 2014; 25: 689-694.

Wang SH, Chou YC, Shangkuan WC, Wei KY, Pan YH, Lin HC. Relationship between Plasma Triglyceride Level and Severity of Hypertriglyceridemic Pancreatitis. PLoS One 2016; 11: e0163984.

Wu BU, Johannes RS, Sun X, Tabak Y, Conwell DL, Banks PA. The early prediction of mortality in acute pancreatitis: a large population-based study. Gut 2008; 57: 1698-1703.

Xiao B, Zhang XM, Jiang ZQ, Tang W, Huang XH, Yang L, Feng ZS. Fatty liver in acute pancreatitis: characteristics in magnetic resonance imaging. J Comput Assist Tomogr 2012; 36: 400-405.

Yadav D. Issues in hyperlipidemic pancreatitis. J Clin Gastroenterol 2003; 36: 54-62.

Yang L, Liu J, Xing Y, Du L, Chen J, Liu X, Hao J. Comparison of BISAP, Ranson, MCTSI, and APACHE II in Predicting Severity and Prognoses of Hyperlipidemic Acute Pancreatitis in Chinese Patients. Gastroenterol Res Pract 2016; $2016: 1834256$.

Zheng Y, Zhou Z, Li H, Li J, Li A, Ma B, Zhang T, Liao Q, Ye Y, Zhang Z, Yang Y. A multicenter study on etiology of acute pancreatitis in Beijing during 5 years. Pancreas 2015; 44: 409-414. 